\title{
EDITORIAL
}

\section{Predictably unpredictable}

\author{
Anahid Basiri \\ School of Geographical and Earth Sciences, The University of Glasgow, Glasgow, UK. \\ E-mail: ana.basiri@glasgow.ac.uk; journal@rin.org.uk
}

Received: 16 May 2021; Accepted: 16 May 2021

Among all quips of the famous American writer, Mark Twain (Samuel Langhorne Clemens), my two favourites are 'Lies, damned lies, and statistics' and 'It is best to read the weather forecasts before we pray for rain'. One may find these two quite paradoxical view towards statistics, prediction, modelling which may remind us of the slippery slope nature of predicting the future. I think we all agree that the covid-19 pandemic has humiliatingly shown us that the future is unpredictable. None of our fancy and intelligent models that have been built on years of data had predicted the recent pandemic at such scale. However, we still use the models to understand the spread of the virus, to predict the results of interventions, and to make science-led decisions and policies. Isn't it ironic? This editorial looks at the paradox of prediction, the certainty of having uncertainty, and the predictability of having an unpredictable future with some brief glance at recent research in the area of navigation.

Let's start with Mark Twain's second statement! Many years ago, when we did not have any knowledge of meteorology, atmospheric laws and statistical forecasting, our ancestors could do nothing but assuming there are randomness and unpredictability in weather so they could only pray for good weather. Later on, we developed our understanding of how climate and atmosphere work improved. We could gather data to produce statistical and numerical weather prediction models. This has allowed us to forecast near-future weather with a relatively acceptable level of accuracy and precision. Obviously, this does not mean we can predict weather conditions for Easter Monday 2078 but for tomorrow early afternoon, one may rely on Met Office models and leave the umbrella at home. However, we have certainly moved on from the time of having little or no knowledge of next week weather, but we still cannot predict so accurately if we look too far ahead.

This pattern, i.e., getting to know phenomena and build a model that can help to understand what is going on while might not be very accurate or useful when it is applied to a different context, far future, exists in many complex and interrelated phenomena. The complexity is the key to the limitations of the models. This complex world can have too many parameters to consider, too many unknowns to get to know, and/or too many interrelated factors and interactions that we either cannot model or simply do not know even exist to be able to consider. For example, navigating autonomous cars in cities is a complex problem, partly because traffic is an unpredictable and complex phenomenon itself, the interactions of the cars with predictably unpredictable humans might be hard to model. On the other hand, launching and navigating a rocket may not be a complex system as almost all forces and features of the rocket are known (up to some acceptable degree of granularity). Of course, rocket science is a very challenging field of research and requires a very deep understanding of physics, math, engineering but the point is modelling the trajectory of a rocket is way more accurate and reliable than the model that can claim the next action of a child jumping on the street "to see how the autonomous car would react"! In the same sense, the human body or stock market are more complex than launching the rocket, which I would like to reiterate is certainly complicated but it is not too complex. There is not a golden algorithm and model that can tell you how our bodies will react to a certain medication, or how the market will behave in a few months' time. What we do know is that almost whenever humans are involved, there are some 
levels of complexity to be added. Humans are a complex breed! But one wonders, if humans are not easy to model, then is the usability of technologies that are used by humans subject to complexity and unpredictability? Who would have thought some people vandalise some infrastructure because they believed there is a link between $5 \mathrm{G}$ and coronavirus?!

Yes! It is difficult to factor in human creativity or to forecast how a piece of technology can be used, misused, vandalised and repurposed. This means there is a limit to what we can say or expect about the future of technologies such as our mobile phone, smart watches and wearables. However, it certainly does not mean we should stop modelling complex phenomena just because there is a limit. The whole of science is about pushing the boundaries and developing our understanding and knowledge of phenomena, even the complex ones. Like the weather forecast example, our models have become much better both in terms of accuracy and also the extent of use. Our knowledge of human mobility, biology and human-computer interaction has extensively improved. And there are better models to help us factor in the interactions between phenomena. Complexity theory is one that is used to construct a holistic model of, for example, human behaviour. Also, we have a better understanding of the parameters and phenomena that play roles in a complex system. Famously Donald Rumsfeld said, 'There are known knowns. These are things we know that we know. There are known unknowns. That is to say, there are things that we know we don't know. But there are also unknown unknowns. There are things we don't know we don't know.' Research helps us to find those hidden unknown unknowns and get to know them or at least make them known unknowns! In this issue of the journal, Trapsilawati et al. (2021) look into the underlying predictors of speeding behaviour and examine the effects of demographic variables on the perceived deterrent mechanisms and predict speeding behaviour to target appropriate prevention programmes. Their paper, which focuses on human behaviour in speeding and driving, looked at 212 randomly selected drivers with valid car driving licences and concluded that demographic variables play an important role in drivers' perceptions towards social and legal sanctions as well as material loss. These findings can be used for policymaking and designing the prevention programmes that prioritise young and single drivers.

Shubina et al. (2021) look at the adoption and efficiency of the digital contact-tracing apps that are used by many countries to control or monitor the covid-19 pandemic. The adoption of digital contact-tracing apps is certainly a good example of a complex challenge. Their paper considers a wide range of factors within three categories of technical, epidemiological and social, and incorporates these into a compact mathematical model. The paper evaluates the effectiveness of digital contact-tracing apps based on received signal strength measurements. The results highlight the limitations, potential and challenges of the adoption of digital contact-tracing apps.

Scagnetto et al. (2021) look into the context-awareness and the end-users of the system in a navigation aid for yacht racing. Mahmoudi et al. (2021) study the challenges of the general airborne camera by modelling the uncertainty and imperfection of the different components. Lens distortion, image delay, rolling shutter, motion blur, interlacing, vignetting, image noise and light level are modelled.

Bao et al. (2021) identify the root causes of young seafarer attrition through semi-structured interviews and questionnaires. The findings of the study show that occupational recognition and family responsibility are the two major factors contributing to outflow of young seafarers. Chinese seafarers' health status is another important factor that has received little attention. In addition, age seems to play a major role in attrition. This paper suggests that a clear career plan could be a potential solution to retain this backbone group as prospective senior officers.

There is a growing push for keeping human in the loop to make sure that data-driven technologies do not blindly over-rule human behaviour and needs. With the rise of new technologies like autonomous cars and unmanned aerial vehicles (UAVs) moving around complex and unpredictable cities and human beings, there is even more need for a better understanding of the parameters and interactions between players of such complex systems. But one thing is always true; there is a limit to what we can predict and to what extent we can predict it but this extent should be always pushed forward. 


\section{References}

Bao, J., Li, Y., Zheng, G. and Zhang, P. (2021). Exploring into contributing factors to young seafarer turnover: Empirical evidence from China. Journal of Navigation, 1-17. doi:10.1017/S0373463321000230

Mahmoudi, A., Sabzehparvar, M. and Mortazavi, M. (2021). A virtual environment for evaluation of computer vision algorithms under general airborne camera imperfections. Journal of Navigation, 1-21. doi:10.1017/S0373463321000060

Scagnetto, I., Brajnik, G., Gus, P. and Trevisan, F. (2021). Oceanus: A context-aware low-cost navigation aid for yacht racing. Journal of Navigation, 1-12. doi:10.1017/S0373463321000205

Shubina, V., Ometov, A., Basiri, A. and Lohan, E. (2021). Effectiveness modelling of digital contact-tracing solutions for tackling the COVID-19 pandemic. Journal of Navigation, 1-34. doi:10.1017/S0373463321000175

Trapsilawati, F., Priatna, N., Wijayanto, T., Widyanti, A., Syafitri, U. and Chamidah, N. (2021). Examining drivers' sociodemographic variables and perceptions towards sanction mechanisms on speeding behaviour on highways: Targeting appropriate prevention. Journal of Navigation, 1-12. doi:10.1017/S0373463321000217 